\title{
Arthroscopic Repair of Acetabular Labral Tears Associated with Femoroacetabular Impingement: 7-10 Years of Long-Term Follow-up Results
}

Jae-Won Lee, MD, Deuk-Soo Hwang, MD, Chan Kang, MD, Jung-Mo Hwang, MD, Hyung-Jin Chung, MD

Department of Orthopaedic Surgery, Chungnam National University School of Medicine, Daejeon, Korea

Background: The purpose of this study is to report the long-term follow-up results of arthroscopic repair of acetabular labral tears with femoroacetabular impingement (FAI).

Methods: Of 45 patients who underwent arthroscopic labral repair under the diagnosis of acetabular labral tears with FAl from January 2008 to December 2010 and met our inclusion criteria, 41 patients who were available for a long-term follow-up were included in the analysis. We compared the long-term follow-up results with the previously reported short-term follow-up results of the same patients. The mean follow-up period was 92.4 months (range, 85 to 117 months). There were 21 males and 20 females, and their mean age at surgery was 34.6 years (range, 16 to 54 years). A modified Harris hip score (mHHS), visual analog scale (VAS), hip outcome score-activity of daily living (HOS-ADL), hip outcome score-activity-sport-specific subscale (HOS-SSS), and patient satisfaction were used for evaluation of the clinical results and Tönnis grade for detection of early osteoarthritis (OA).

Results: The mean VAS score decreased from 6.4 points to 1.8 points $(p<0.001)$, the mean mHHS increased from 59.5 points to 86.8 points $(p<0.001)$, and the mean HOS-ADL and HOS-SSS increased from 58.3 and 51.2 , respectively, to 85.2 and 82.4 , respectively $(p<0.001)$, between the preoperative and last follow-up assessment. The mean patient satisfaction score was 7.6 of 10 . The average Tönnis grade at the last follow-up (0.67; range, 0 to 3 ) was not significantly different from the preoperative average (0.51; range, 0 to 1). Only one case was converted to total hip arthroplasty because of progression of $O A$ at 8 years after surgery. Five cases of secondary arthroscopic surgery were performed before maximum 5 years postoperatively because of labro-synovial adhesion (three cases), pullout of the suture anchor (one case) or symptomatic heterotrophic ossification (one case).

Conclusions: The clinical and radiological long-term follow-up revealed that improvement after arthroscopic labral repair and osteoplasty for FAl were maintained in most cases without significant progression of arthritis. Anatomical recovery of the acetabular labrum was associated with the improvement of clinical symptoms.

Keywords: Hip, Labral tear, Femoroacetabular impingement, Arthroscopy, Labral repair

The acetabular labrum is a triangular fibrocartilaginous tissue located between the acetabulum articular cartilage and the joint capsule, which increases the stability of the

Received August 30, 2018; Accepted October 29, 2018

Correspondence to: Deuk-Soo Hwang, MD

Department of Orthopaedic Surgery, Chungnam National University

School of Medicine, 266 Munhwa-ro, Jung-gu, Daejeon 35015, Korea

Tel: +82-42-338-2480, Fax: +82-42-338-2482

E-mail: dshwang@cnu.ac.kr hip joint. ${ }^{1)}$ Inside the acetabular labrum, there are nerve endings involved in proprioception and nociception; thus, pain can occur when the labrum is damaged. ${ }^{2)}$ It was reported that pathological changes in the acetabular labrum lead to chronic acetabular pain and limited quality of life. ${ }^{3,4)}$ In the past, Altenberg ${ }^{3)}$ also suggested labral tears as a cause of hip joint pain not related to trauma in 1977. He reported that severe pain and mechanical symptoms occurred due to a translocated acetabular labrum and symptomatic relief could be obtained immediately after 
Lee et al. Arthroscopic Labral Repair

Clinics in Orthopedic Surgery • Vol. 11, No. 1, 2019•www.ecios.org

removal of the acetabular labrum. However, once resected, the labrum is not regenerated; thus, the role of acetabular labrum in the hip joint has been extensively studied. Recently, there has been remarkable improvement in hip arthroscopy instruments and skills related to labral tears. In addition, the acetabular labral tear has become of great interest due to its relation with hip arthritis. ${ }^{5)}$

It has been reported that arthroscopic acetabular labral resection, effective for immediate pain relief, could damage the inherent physiological function of the labrum. $^{6-9)}$ There is no literature on the long-term outcome of labral repair in hip arthroscopy especially in the Asian population. The purpose of this study was to analyze the long-term follow-up results of arthroscopic acetabular labral repair and to determine the effectiveness of labral repair in Asian femoroacetabular impingement (FAI) patients. $^{10-12)}$

\section{METHODS}

We conducted this study in compliance with the principles of the Declaration of Helsinki. The protocol of this study was reviewed and approved by the Institutional Review Board of Chungnam National University Hospital Institutional Review Board (IRB No. CNUH 2018-08-032). All of the patients provided informed consent.

\section{Patient Selection}

A retrospective review of all cases that underwent hip arthroscopy at our institution from January 2008 to December 2010 was performed. The procedure was performed by the same senior surgeon (DSH) in all cases. Only patients with complete medical records including operation records, follow-up scores and preoperative, postoperative and follow-up radiographs were included. Patients who had the following hip conditions previously were excluded: Legg Calves-Perthes disease, infectious hip disease, avascular necrosis, rheumatic disease, pyogenic and tuberculous arthritis diffuse idiopathic skeletal hyperostosis, ankylosing spondylitis, and severe dysplasia. Patients with preoperative Tönnis osteoarthritis (OA) grade 2 or more were also excluded. A total of 45 patients were found to satisfy the inclusion and exclusion criteria. Additional three-dimensional computed tomography (3D-CT) and magnetic resonance imaging (MRI) were performed in patients with chronic hip pain suspected of an acetabular labral tear based on medical history taking, physical examination, and plain radiography.

History taking performed during the first visit three included the onset, cause and aggravation of pain.
Physical examinations such as log rolling, Patrick test and impingement test were performed. ${ }^{13,14)}$ Simple radiography was performed to obtain the pelvic anteroposterior view, frog-leg side view, false-profile view and cross-table view to confirm the acetabular or femoral bony deformities, femoral-acetabular dysplasia and bump formation in the femoral neck. In addition, 3D-CT and MRI were performed to check bony deformities and labral pathologies. All the radiographs were evaluated by two orthopedic surgeons (JWL and JMH) and one orthopedic radiologist (STK) working at the same hospital. Because of the low sensitivity of MRI for detection of labral tears, we did not depend on MRI but relied on physical examinations and duration of symptoms to determine surgical indications. Hip arthroscopy was performed under general or epidural anesthesia. Arthroscopic findings of labral tears were classified with respect to radial fibrillation, peripheral longitudinal tears, bucket-handle tears, complete detachment, atrophy or absence and mixed type according to the classification of Lage et al. ${ }^{15)}$ FAI was confirmed by checking impingement during hip motions in arthroscopy.

There were 22 males and 23 females, and their mean age at the time of surgery was 33.0 years (range, 16 to 54 years). The lesion was located on the right side in 28 cases (62\%) and the left side in 17 cases (38\%). Of the 45 patients, four patients (one male and three females) were not available for a long-term follow-up. Thus, the remaining 41 cases followed up for 85-117 months (mean, 92.4 months) were reviewed for analysis. Ultimately, patients included consisted of 21 males and 20 females with a mean age of 34.6 years (range, 16 to 54 years). The mean body

\section{Table 1. Demographic Data}

Variable

Value (41 hips)

\begin{tabular}{lc} 
Sex & \\
\hline Male & 21 \\
\hline Female & 20 \\
\hline Side & 26 \\
\hline Right & 15 \\
\hline Left & 34.6 \\
\hline Age at surgery $(\mathrm{yr})$ & $24.3 \pm 3.3(18.6-38.5)$ \\
\hline Body mass index $\left(\mathrm{kg} / \mathrm{m}^{2}\right)$, mean \pm SD (range) & $92.4(85-117)$ \\
\hline Follow-up time $(\mathrm{mo})$, mean (range) & 91 \\
\hline Follow-up rate $(\%)$ & $2 \%$ \\
\hline
\end{tabular}

SD: standard deviation. 
mass index was $24.3 \pm 3.3 \mathrm{~kg} / \mathrm{m}^{2}$ (range, 18.6 to $38.5 \mathrm{~kg} / \mathrm{m}^{2}$ ). Patient demographics are summarized in Table 1.

\section{Surgical Technique and Postoperative Care}

Standard portals were used with the patient lying on the fracture table. First, capsulotomy was performed between the anterior and anterolateral parts of the femur neck using an arthroscopic knife (Banana blade, $254 \mathrm{~mm}$; Arthrex, Singapore) to handle the arthroscope easily. After confirming the lesion through arthroscopy, acetabular cartilage debridement was performed and suture was performed using a nonabsorbable suture anchor as descried by Kelly et al. ${ }^{16)}$ Labral repair was achieved with sutures looped around the torn segments or placed through the torn labrum (looped stitch technique) and performed usually using 2-3 stitches for each torn labrum at $1-\mathrm{cm}$ intervals. Each suture was anchored to the acetabular rim, with the suture anchor $(2.3 \mathrm{~mm}$ Osteoraptor; Smith \& Nephew, Andover, MA, USA) placed approximately 2 to $3 \mathrm{~mm}$ above the cartilage surface. Then, femoroplasty was performed in the femoral neck after flexion of the hip if a bump formed at the femoral head-neck junction.

From the first postoperative day, the pendulum movement of the hip joint and partial weight-bearing us-
A

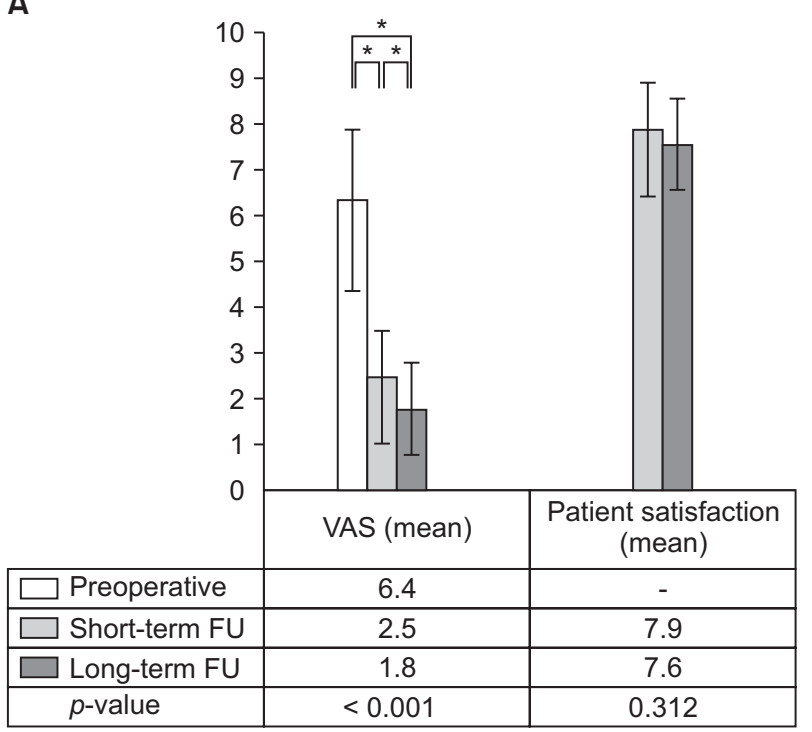

C

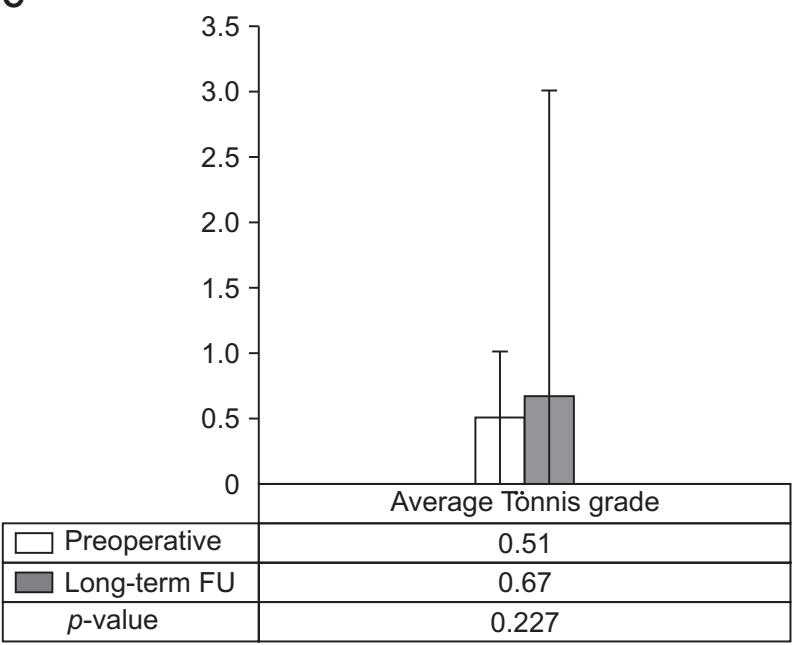

B

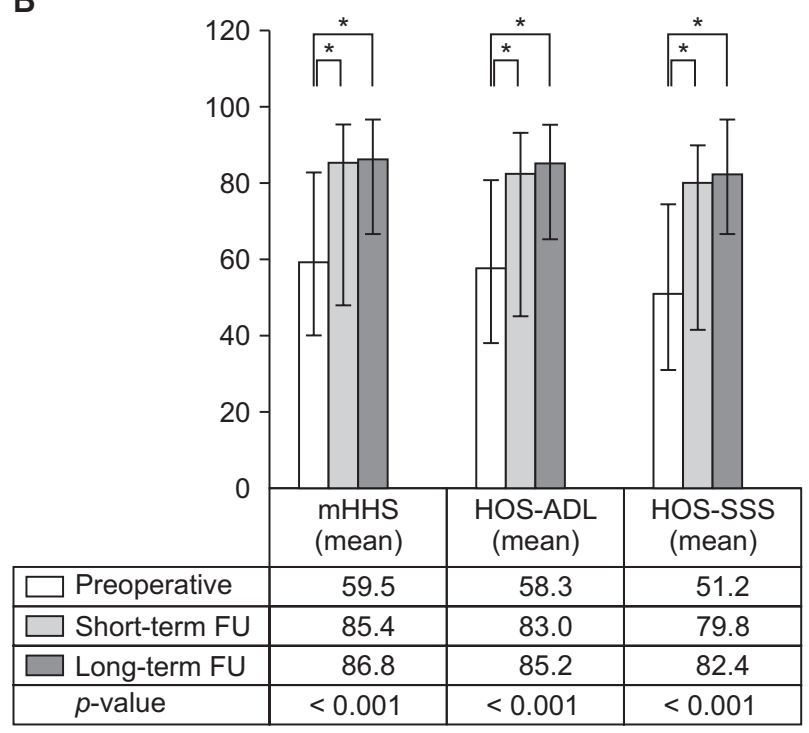

D

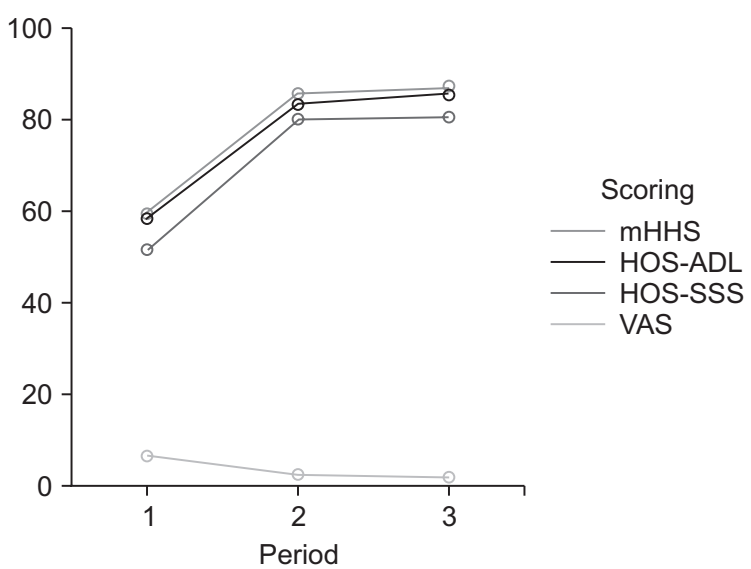

Fig. 1. Visual analog scale (VAS) score and patient satisfaction score (A) and patient-reported outcome (B) collected preoperatively, at the short-term follow-up (FU; 2-5 years), and at the long-term FU (7-10 years). (C) Average Tönnis grade measured preoperatively and at the last long-term FU. (D) The graph shows changes in scores by the period (1: preoperative, 2: short-term FU, 3: long-term FU). mHHS: modified Harris hip score, HOS-ADL: hip outcome score-activity of daily living, HOS-SSS: hip outcome score-sport-specific subscale. *Scheffe's post hoc analysis, $p<0.005$. 
ing crutches were allowed, but more than $90^{\circ}$ of hip flexion motion was prohibited. Around three days after the operation, the patient was discharged and follow-up in the outpatient clinic to monitor the clinical outcome. Full weight-bearing of the hip joint was allowed 4 weeks after the operation.

\section{Clinical Outcomes Measurement and Statistical Analyses} Clinical and radiographic follow-up evaluations were performed at 6 weeks; 3, 6 and 12 months; and every 1 year thereafter. Patients who did not attend regularly scheduled visits were contacted by telephone. Clinical assessment was performed using a modified Harris hip score (MHHS), visual analog scale (VAS), hip outcomes score (HOS), and patient satisfaction score (0-10 scale; 10 , very satisfied). The last long-term follow-up clinical results were compared with preoperative values and short-term follow-up results. Tönnis grade was classified by simple radiological changes. Statistical analysis was conducted using repeated measures analysis of variance test with Scheffe's post hoc test for MHHS, VAS and HOS and a paired $t$-test for Tönnis grade in IBM SPSS ver. 21.0 (IBM Corp., Armonk, NY, USA). A $p$-value $<0.05$ was considered statistically significant for all differences.

\section{RESULTS}

For evaluation of clinical outcomes, preoperative values, short-term (2-5 years) follow-up results and last longterm follow-up (7-10 years) results were compared. The mean VAS score significantly improved from 6.4 points (range, 2 to 9 points) preoperatively to 2.5 points (range, 0 to 7 points) at the short-term follow-up, and then to 1.8 points at the last long-term follow-up $(p<0.01)$. The mean mHHS score improved from 59.5 (range, 37.5 to 82 ) preoperatively to 85.4 (range, 60 to 94.6 ) in short-term and then to 86.8 (range, 61 to 95.7) in long-term follow-up $(p<0.01)$. Activities of daily living HOS was significantly improved from $58.3 \%$ (range, $20 \%$ to $80 \%$ ) preoperatively to $83.0 \%$ (range, $45 \%$ to $95 \%$ ) in the short-term and then to $85.2 \%$ (range, $45 \%$ to $95 \%$ ) in the long-term follow-up ( $p$ $<0.01$ ); sports-related activities of HOS was also improved from $51.2 \%$ (range, $10 \%$ to $80 \%$ ) to $79.8 \%$ (range, $50 \%$ to $95 \%$ ) and then to $82.4 \%$ (range, $50 \%$ to $95 \%$; $p<0.01$ ) in the respective period. At the last follow-up, the mHHS was more than 80 points (satisfied or very satisfied) in 35 cases $(78.8 \%)$ and less than 70 points (dissatisfaction) in three cases $(6.7 \%)$. The mean patient satisfaction score was $7.6 \pm$ 2.2. The average Tönnis grade based on the last follow-up simple radiography was 0.67 (range, 0 to 3 ), which showed no statistically significant difference from the initial average grade of 0.51 (range, 0 to $1 ; p=0.227$ ) (Fig. 1).
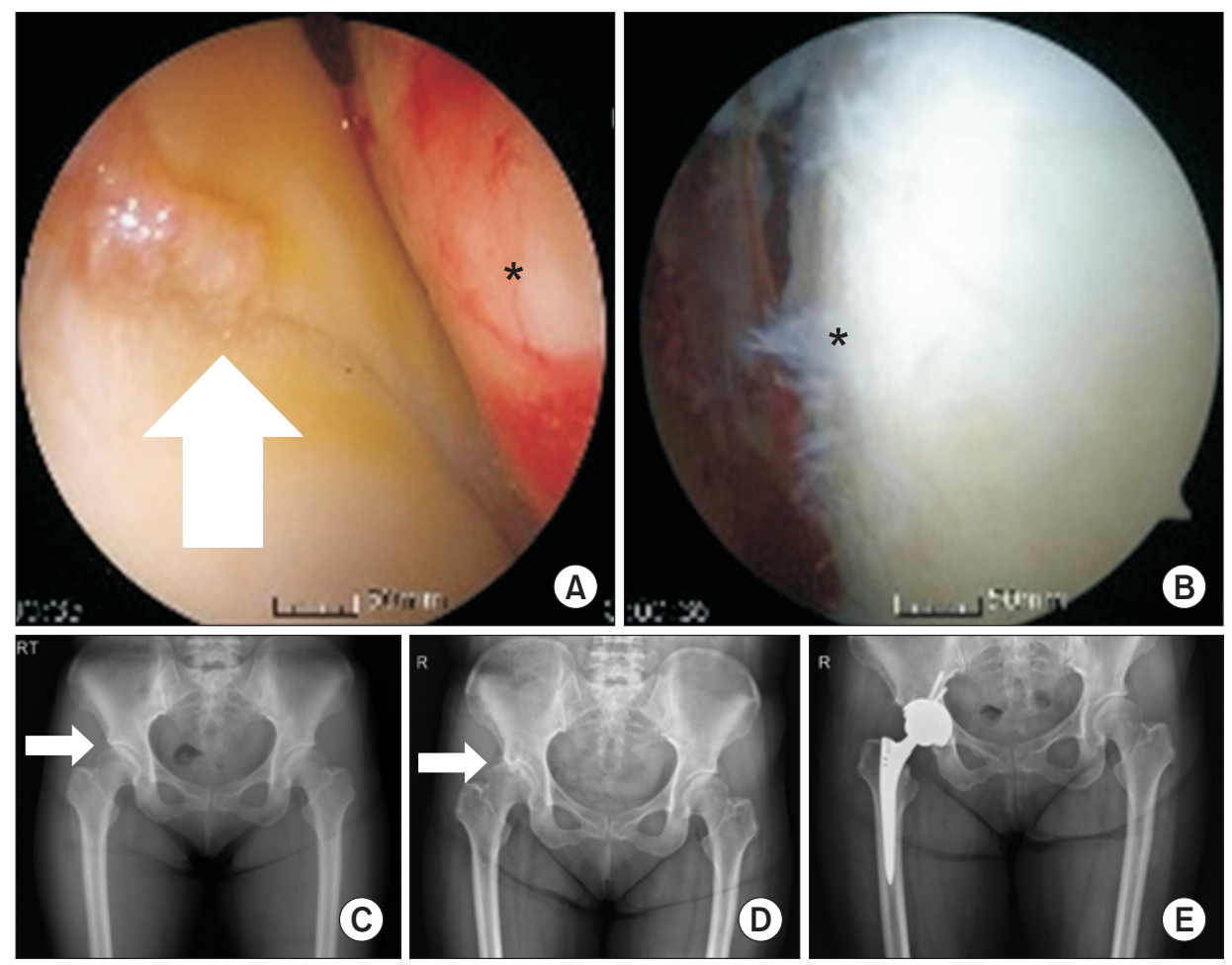

Fig. 2. A 47-year-old female had a right hip femoroacetabular impingement and acetabular labral tear. She underwent arthroscopic labral repair using two anchors and arthroscopic bumpectomy. (A) In arthroscopy, early degenerative change was shown in the femoral head cartilage (asterisk) and labral surface (arrow). (B) Acetabular cartilage surface fibrillation (asterisk) was observed. (C) She had a mild dysplastic hip on the right side and a Tönnis grade 1 osteoarthritis (arrow) in the preoperative period. (D) Joint space narrowing and degenerative changes developed gradually and Tönnis grade 3 (arrow) osteoarthritis was found at the last 86-month follow-up. (E) She received total hip arthroplasty. 
Lee et al. Arthroscopic Labral Repair

Clinics in Orthopedic Surgery • Vol. 11, No. 1, 2019•www.ecios.org

Table 2. Revisional Arthroscopic Procedure and Conversion to THA

\begin{tabular}{lccc}
\multicolumn{1}{c|}{ Variable } & Total & $\begin{array}{c}\text { Short-term follow-up } \\
\text { (2-5 yr) }\end{array}$ & $\begin{array}{c}\text { Long-term follow-up } \\
\text { (after } 7 \text { yr) }\end{array}$ \\
\hline Revisional arthroscopic surgery & $5(12.2)$ & $5(12.2)$ & 0 \\
\hline Conversion to THA & $1(2.4)$ & 0 & $1(2.4)$ \\
\hline Time to revisional arthroscopic surgery (mo) & 26.6 & $26.6(15-49)$ & - \\
\hline Time to THA conversion (mo) & 86 & 0 & 86 \\
\hline
\end{tabular}

Values are presented as number (\%) or mean (range).

THA: total hip arthroplasty.

Six patients (14.6\%) underwent reoperation due to complications. No other complications were noted. Five cases (12.2\%) underwent revisional arthroscopic procedures and one case (2.4\%) had conversion to total hip arthroplasty (THA). Secondary arthroscopic procedures were performed at a mean of 26.6 months after surgery (range, 15 to 49 months). There were three cases of labrosynovial adhesions due to synovial hyperplasia in the acetabular region, one case of pullout of the suture anchor and one case of heterotopic ossification. The one case of conversion to THA occurred at 86 months postoperatively. The Tönnis grade was 1 preoperatively in the patient, but due to joint space narrowing and degenerative changes, the grade at the last 86 months of follow-up was 3, which required THA (Fig. 2). Data on all revisional arthroscopic procedures and conversion to THA are summarized in Table 2.

\section{DISCUSSION}

Larson et al. ${ }^{11)}$ compared the arthroscopic results of debridement and labral repair in cases of FAI and observed better results with repair. The mean mHHS of the repair group was 64.5 before surgery and 94.3 in the final evaluation, which was 9.4 points higher than that in the debridement group. Jackson et al. ${ }^{17)}$ evaluated 54 patients who had undergone arthroscopic labral repair. They observed significant improvement in the four scores at 2.4 years after the procedure; the mean mHHS was 63.7 preoperatively and 89.9 at the final evaluation. Furthermore, $85.2 \%$ of the patients had good or excellent results. Similarly, three functional scores, VAS and satisfaction were used in our study and we also had favorable results.

Repair of labral tears is preferred than resection of labral tears for preservation of the function of the joint. Many clinical outcome studies have provided supporting biomechanical data and conclusions. Multiple compara- tive cohort studies and one prospective randomized study have demonstrated that patients undergoing labral repair have significantly better outcomes than labral debridement. ${ }^{11,18,19)}$ The systematic review by Ayeni et al., ${ }^{20)}$ which includes most of these studies as well as the outcomes of open procedures, found similar results favoring repair over debridement. Other recent studies also discovered labral debridement procedures caused micromotions in the hip joint, contributing to the development of OA. ${ }^{21)}$ With these reports in mind, we repaired acetabular labrum tears whenever possible, considered that the labrum is essential for joint-cartilage protection.

Arthroscopic labral repair outcomes in our study were favorable. Compared with the short-term results in the same patient group published 5 years ago, ${ }^{22)}$ the VAS, mHHS, HOS were not improved significantly at the longterm follow-up. Scheffe's post hoc test revealed that the mHHS and HOS more significantly improved during the short-term follow-up $(p<0.05)$. Of note, however, is that there was improvement in outcomes in a slow and continuous manner. On the other hand, improvement in clinical outcomes of the arthroscopic labral resection group published previously by our study team ${ }^{23)}$ was not the same. The mean mHHS in this group was 58.9 preoperatively, 81.9 in the short-term follow-up and 79.3 at the final follow-up. Similarly, the hip outcome score-activity of daily living (HOS-ADL) and sport-specific subscale (HOS-SSS) that improved in the short-term period slightly decreased at the final mid-term follow-up. So labral repair procedures could be more conducive to long-term improvement of the patient's functional scores than labral resection procedures.

There are not many studies on the long-term results of arthroscopic operation for FAI. Menge et al. ${ }^{24)}$ reported 10-year outcomes and the hip survival rate of hip arthroscopy for FAI to compare labral resection with labral repair. In the study, significant improvement in 
clinical outcomes (HOS-ADL, HOS-SSS, mHHS, short form 12 health survey and physical composite scores) was obtained regardless of the labral procedure. Seven of the 154 patients (4.5\%) underwent revisional hip arthroscopy, two of whom were in the labral resection group and the other were in the labral repair group. They reported that $34 \%$ of the patients had a THA within 10 years following the arthroscopy and the rate of conversion to THA was not related to the labral treatment method. Our study is a case series report of a labral repair group, so we could not compared with a labral resection group. However, the significance of this study can be found in the fact that there have been no other studies in Asia reporting the long-term follow-up outcomes in FAI.

Except for the case that required conversion THA, all revisional arthroscopic procedures were performed in the short-term follow-up period. There was no case that required a revisional arthroscopic procedure in the midterm and long-term follow-up periods. Therefore, it could be interpreted that the hip joint is more stabilized after a short-term period following arthroscopic labral repair.

In this study, the most common cause of second- look hip arthroscopy was incomplete osteoplasty for FAI. ${ }^{25}$ Incomplete osteoplasty means that the femoral neck junction is less shaved than the length of the burr of $5 \mathrm{~mm}$, and the femoral head is hooked to the acetabular labrum when the hip is flexed $70^{\circ}-80^{\circ}$. It can be also assessed with postoperative radiographs where the alpha angle is $>55^{\circ}$ and offset is $\leq 9 \mathrm{~mm}$. In cases of labro-synovial adhesion in revisional arthroscopic procedures, incomplete femoroplasty with an alpha angle of $64.5^{\circ}$ and offset of $7.8 \mathrm{~mm}$ was shown in radiographs after primary arthroscopic procedure; this, it is considered that complete femoroplasty may be important to prevent labro-synovial adhesion (Fig. 3).

Several studies have described failure rates of labral tear repair. Gupta et al. ${ }^{26)}$ reported a $9 \%$ conversion to THA following hip arthroscopy. Sawyer et al. ${ }^{27)}$ reported the rate of conversion to THA was $2 \%$ in patients with $>2$ $\mathrm{mm}$ of joint space who underwent labral repair. Harris et al. ${ }^{28)}$ reported a $2.9 \%$ conversion to THA following hip arthroscopy. Menge et al. ${ }^{24)}$ reported that $34 \%$ of patients had conversion to THA due to $<2 \mathrm{~mm}$ of joint space preoperatively. On the other hand, our THA conversion rate (2.4\%) was very low compared to others. In our THA conversion
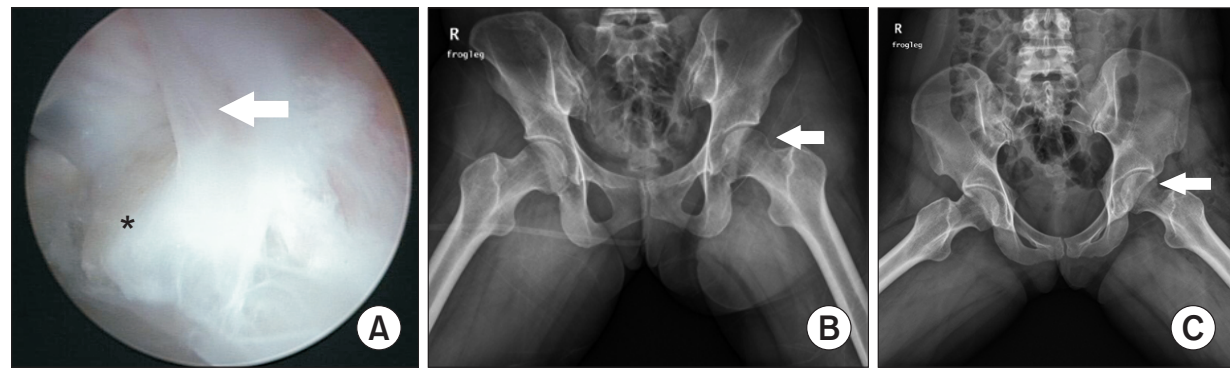

Fig. 3. A 28-year-old female received arthroscopic labral repair and femoroplasty. After 2 months, she received revisional hip arthroscopy for pain and limited motions. (A) In arthroscopy, severe adhesion between the labrum (asterisk) and hip joint synovial capsule (arrow) was observed. (B) The simple frog-leg radiograph after primary arthroscopy showed incomplete femoroplasty on the femoral neck area (arrow) where the alpha angle was $64.5^{\circ}$ and offset was $7.8 \mathrm{~mm}$. (C) On the final frog-leg simple radiograph, we could confirm complete femoroplasty was performed: the alpha angle was $49.5^{\circ}$ and offset was $10.2 \mathrm{~mm}$ (arrow) after revisional arthroscopy.
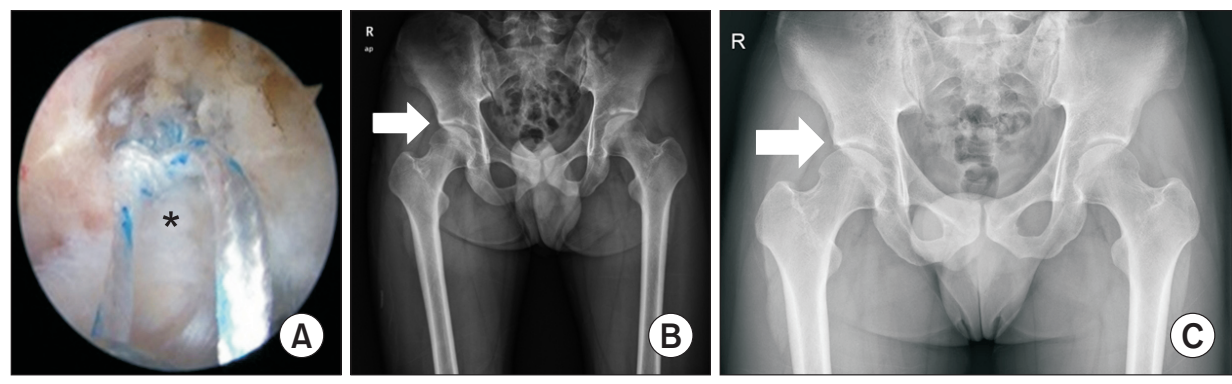

Fig. 4. (A) A 21 -year-old male received arthroscopic labral repair (asterisk) and bumpectomy because of femoroacetabular impingement and labral tear in the right hip joint. (B) Preoperative simple radiogrph showing Tönnis grad 0 osteoarthritis (arrow) in the preoperative period. (C) Eight years later, the joint space became wider as he grew and the Tönnis grade was not changed (arrow). 
case, the patient already had early osteoarthritic changes (Tönnis grade 1) with cartilage defect and had a borderline dysplasia of the hip where the lateral center edge angle was $24.8^{\circ}$, which could have affected the development and progression of OA. Unlike other studies, we narrowed indications for arthroscopic treatment. Only those with normal or below Tönnis grade 1 were included, which could be the reason to have a lower THA conversion rate in our study.

Except the one THA conversion case, other cases did not have significant changes in the Tönnis grade in the long-term follow-up (Fig. 4). Therefore, it could be estimated that the arthroscopic labral repair technique could help stabilize the hip joint and delay progression of osteoarthritic changes in the long-term follow-up. Additional revision arthroscopic procedures and conversion to THA were performed, but the survivorship rate remained above $85 \%$ at the last follow-up. Theses outcomes demonstrated the effectiveness of the arthroscopic labral repair technique.

Our study has a few limitations. First of all, this study does not have a control or comparison group who underwent labral debridement or conservative therapy. Second, after arthroscopic labral repair, we educated the patients on life style modifications (downgrade for activities and exercise sports). That may also be related to the improvement in clinical scores and delay of the progression of OA. Last, we performed not only labral repair but also femoroplasty or acetabuloplasty, synovectomy, ligamentum teres debridement and so on. In particular, femoroplasty or acetabuloplasty could affect clinical results during the follow-up because it is effective for improving range of motion and decreasing symptomatic pain caused by impingement.

The clinical and radiological long-term follow-up revealed that most cases of arthroscopic management of FAI and labral tears maintained improvement of outcome without progression of significant arthritis. Because of the importance of the main function of the labrum, we recommend labral repair for symptomatic acetabular labral tears. Anatomical recovery of acetabular labrum was associated with the improvement of clinical symptoms.

\section{CONFLICT OF INTEREST}

No potential conflict of interest relevant to this article was reported.

\section{ACKNOWLEDGEMENTS}

This research was supported by Chungnam National University Hospital Research Fund 2017 in Daejeon, Korea.

We were assisted by Soon-Tae Kwon in Department of Radiology, Chungnam National University Hospital.

\section{REFERENCES}

1. Takechi $\mathrm{H}$, Nagashima H, Ito S. Intra-articular pressure of the hip joint outside and inside the limbus. Nihon Seikeigeka Gakkai Zasshi. 1982;56(6):529-36.

2. Kim YT, Azuma H. The nerve endings of the acetabular labrum. Clin Orthop Relat Res. 1995;(320):176-81.

3. Altenberg AR. Acetabular labrum tears: a cause of hip pain and degenerative arthritis. South Med J. 1977;70(2):174-5.

4. Czerny C, Hofmann S, Neuhold A, et al. Lesions of the acetabular labrum: accuracy of MR imaging and MR arthrography in detection and staging. Radiology. 1996;200(1):22530 .

5. McCarthy JC, Noble PC, Schuck MR, Wright J, Lee J. The Otto E. Aufranc Award: the role of labral lesions to development of early degenerative hip disease. Clin Orthop Relat Res. 2001;(393):25-37.

6. Ferguson SJ, Bryant JT, Ganz R, Ito K. The influence of the acetabular labrum on hip joint cartilage consolidation: a poroelastic finite element model. J Biomech. 2000;33(8):953-
60.

7. Ferguson SJ, Bryant JT, Ganz R, Ito K. The acetabular labrum seal: a poroelastic finite element model. Clin Biomech (Bristol, Avon). 2000;15(6):463-8.

8. Ferguson SJ, Bryant JT, Ganz R, Ito K. An in vitro investigation of the acetabular labral seal in hip joint mechanics. J Biomech. 2003;36(2):171-8.

9. Petersen W, Petersen F, Tillmann B. Structure and vascularization of the acetabular labrum with regard to the pathogenesis and healing of labral lesions. Arch Orthop Trauma Surg. 2003;123(6):283-8.

10. Espinosa N, Rothenfluh DA, Beck M, Ganz R, Leunig M. Treatment of femoro-acetabular impingement: preliminary results of labral refixation. J Bone Joint Surg Am. 2006;88(5):925-35.

11. Larson CM, Giveans MR, Stone RM. Arthroscopic debridement versus refixation of the acetabular labrum associated with femoroacetabular impingement: mean 3.5-year follow- 
Lee et al. Arthroscopic Labral Repair

Clinics in Orthopedic Surgery • Vol. 11, No. 1, 2019•www.ecios.org

up. Am J Sports Med. 2012;40(5):1015-21.

12. Philippon MJ, Ejnisman L, Ellis HB, Briggs KK. Outcomes 2 to 5 years following hip arthroscopy for femoroacetabular impingement in the patient aged 11 to 16 years. Arthroscopy. 2012;28(9):1255-61.

13. Klaue K, Durnin CW, Ganz R. The acetabular rim syndrome: a clinical presentation of dysplasia of the hip. J Bone Joint Surg Br. 1991;73(3):423-9.

14. Suenaga E, Noguchi Y, Jingushi S, et al. Relationship between the maximum flexion-internal rotation test and the torn acetabular labrum of a dysplastic hip. J Orthop Sci. 2002;7(1):26-32.

15. Lage LA, Patel JV, Villar RN. The acetabular labral tear: an arthroscopic classification. Arthroscopy. 1996;12(3):269-72.

16. Kelly BT, Weiland DE, Schenker ML, Philippon MJ. Arthroscopic labral repair in the hip: surgical technique and review of the literature. Arthroscopy. 2005;21(12):1496-504.

17. Jackson TJ, Hanypsiak B, Stake CE, Lindner D, El Bitar YF, Domb BG. Arthroscopic labral base repair in the hip: clinical results of a described technique. Arthroscopy. 2014;30(2):208-13.

18. Philippon MJ, Briggs KK, Yen YM, Kuppersmith DA. Outcomes following hip arthroscopy for femoroacetabular impingement with associated chondrolabral dysfunction: minimum two-year follow-up. J Bone Joint Surg Br. 2009;91(1):16-23.

19. Krych AJ, Thompson M, Knutson Z, Scoon J, Coleman $\mathrm{SH}$. Arthroscopic labral repair versus selective labral debridement in female patients with femoroacetabular impingement: a prospective randomized study. Arthroscopy. 2013;29(1):46-53.

20. Ayeni OR, Adamich J, Farrokhyar F, et al. Surgical management of labral tears during femoroacetabular impingement surgery: a systematic review. Knee Surg Sports Traumatol
Arthrosc. 2014;22(4):756-62.

21. Song Y, Ito H, Kourtis L, Safran MR, Carter DR, Giori NJ. Articular cartilage friction increases in hip joints after the removal of acetabular labrum. J Biomech. 2012;45(3):52430.

22. Jeon YS, Hwang DS, Kang C, Hwang JM, Lee GS. Arthroscopic labral repair associated with femoroacetabular impingement: short term 2-5 years follow-up results. Hip Pelvis. 2013;25(2):115-20.

23. Kang C, Hwang DS, Jeon YS, Han SC, Lee GS, Kang DH. Arthroscopic treatment of femoroacetabular impingement of the hip: 5-7 years result. Hip Pelvis. 2012;24(3):237-44.

24. Menge TJ, Briggs KK, Dornan GJ, McNamara SC, Philippon MJ. Survivorship and outcomes 10 years following hip arthroscopy for femoroacetabular impingement: labral debridement compared with labral repair. J Bone Joint Surg Am. 2017;99(12):997-1004.

25. Hwang DS, Kang C, Cha SM, Kim JH. Second-look hip arthroscopy after arthroscopic labrectomy of the hip: preliminary report. J Korean Orthop Assoc. 2009;44(4):480-5.

26. Gupta A, Redmond JM, Stake CE, Dunne KF, Domb BG. Does primary hip arthroscopy result in improved clinical outcomes?: 2-year clinical follow-up on a mixed group of 738 consecutive primary hip arthroscopies performed at a high-volume referral center. Am J Sports Med. 2016;44(1):74-82.

27. Sawyer GA, Briggs KK, Dornan GJ, Ommen ND, Philippon MJ. Clinical outcomes after arthroscopic hip labral repair using looped versus pierced suture techniques. Am J Sports Med. 2015;43(7):1683-8.

28. Harris JD, McCormick FM, Abrams GD, et al. Complications and reoperations during and after hip arthroscopy: a systematic review of 92 studies and more than 6,000 patients. Arthroscopy. 2013;29(3):589-95. 